DR. ALEX ROSSOR (Orcid ID : 0000-0003-4648-2896)

Article type : Editorials

\title{
Broadening the genetic spectrum of distal hereditary motor neuropathy
}

\section{Alexander M Rossor}

Queen Square Centre for Neuromuscular Diseases. UCL Institute of Neurology and National Hospital for Neurology and Neurosurgery, London, WC1N 3BG, UK

Email: a.rossor@ucl.ac.uk

In this issue of the Journal, Frasquet et al. report on the genetic spectrum of a large cohort of patients with distal hereditary motor neuropathy ( $\mathrm{dHMN}$ ) from two large centres in Spain ${ }^{1}$. All patients underwent detailed clinical assessment by experts in neuromuscular disease and are likely to have an accurate diagnosis. As the centre in Valencia provides all neuromuscular services for a defined geographical area, these two factors have allowed the authors to calculate a population prevalence for dHMN. The figure they calculate of 1 in 50,000 is similar to other studies from the North of England ${ }^{2}$.

All patients, except those with a historical genetic diagnosis, underwent sequencing of either the whole exome or one of four comprehensive neuropathy gene panels. All panels included most dHMN genes with a few exceptions (MYH14 was not included in one of the Valencia panels, AAAS was not included in the Valencia panels and WARS was not in any of the panels). All panels included the familial ALS genes TARDP, FUS, SOD, VCP, VAPB and HNRNPA1. Although the authors identified a small number of patients with the E22G SOD1 mutation, the absence of mutations in any of the other ALS genes is perhaps more noteworthy. SOD1 ALS causes a predominantly lower motor neuron syndrome and unlike many of the other genes, there is no phenotypic overlap with frontotemporal dementia ${ }^{3}$. The C9orf72 expansion was not tested, but even allowing for this, there does not appear to be significant genetic overlap between ALS and dHMN.

The diagnostic rate in this cohort of patients was significantly different between familial and sporadic cases. For familial index cases the diagnostic rate was $67.4 \%$ whereas for sporadic cases this was only $12.3 \%$. In the Valencia cohort, $60 \%$ of genetically confirmed cases were autosomal dominant and $40 \%$ recessive and This article has been accepted for publication and undergone full peer review but has not been through the copyediting, typesetting, pagination and proofreading process, which may lead to differences between this version and the Version of Record. Please cite this article as doi: 10.1111/ENE.14734

This article is protected by copyright. All rights reserved 
in the Barcelona cohort the split was $50 \%$. The Valencia population is known to have a high level of consanguinity and so the genetic spectrum in this study may not be reflective of other European states. Despite the high level of consanguinity, it is noteworthy that SORD mutations only accounted for $3 \%$ of $\mathrm{dHMN}$ cases despite a carrier frequency of the commonest mutation of 1 in $3000^{4}$. This may be partly attributable to the frequent sensory involvement in SORD neuropathy that may have excluded affected individuals from inclusion in this study.

The significant difference in the diagnostic rate between familial and sporadic cases raises the possibility that some sporadic cases may not have dHMN. This is especially true if the motor neuropathy develops in middle to late adulthood when acquired causes are more common. However, this does not fully explain the picture as the age group with the highest proportion of genetically undiagnosed cases were those patients with disease onset in the first and second decades of life. As it is unusual for $\mathrm{dHMN}$ in isolation to affect fertility, this suggests that many of the undiagnosed cases are likely to be due to recessive rather than de novo dominant mutations. Complex genomic rearrangements known to cause dHMN were not screened for in this population but are likely to account for only a small number of the patients ${ }^{5}$.

The Barcelona panel in the study included the AAAS gene, mutations in which cause 'Triple A syndrome', a complex disease consisting of achalasia, alacrima, adrenal insufficiency and a peripheral neuropathy. Mutations in AAAS are not traditionally considered to cause pure dHMN, however in some of the patients in this study, a distal motor neuropathy was the presenting feature. This is an important finding for two reasons. Firstly, it suggests that $A A A S$ should be included in neuropathy gene panels and secondly that other complex syndromes with a motor predominant neuropathy (e.g. SCA2) may explain some genetically undiagnosed patients with dHMN.

Finally, one of the most important lessons from this study is the importance of segregation analysis for determining the pathogenicity of Variants of Uncertain Significance (VUS). Of the 37 VUSs identified, segregation analysis allowed the reclassification of $50 \%$ of VUS variants as benign. With odds such as these, caution should be exercised for attributing clinical significance to any VUS in which segregation analysis is not possible.

\section{References}

1. Frasquet. No Title. Eur J Neurol. 2021.

2. Bansagi B, Griffin H, Whittaker RG, et al. Genetic heterogeneity of motor neuropathies. Neurology. 2017;88(13):1226-1234. doi:10.1212/WNL.0000000000003772.

3. Syriani E, Morales M, Gamez J. The P.E22G mutation in the Cu/Zn superoxide-dismutase gene predicts a long survival time. J Neurol Sci. 2009;285(1-2):46-53. doi:10.1016/j.jns.2009.05.011.

4. Cortese A, Zhu Y, Rebelo AP, et al. Biallelic mutations in SORD cause a common and potentially treatable hereditary neuropathy with implications for diabetes. Nat Genet. 2020;52(5):473-481.

This article is protected by copyright. All rights reserved 
doi:10.1038/s41588-020-0615-4.

5. Drew AP, Cutrupi AN, Brewer MH, Nicholson GA, Kennerson ML. A 1.35 Mb DNA fragment is inserted into the DHMN1 locus on chromosome 7q34-q36.2. Hum Genet. 2016;135(11):1269-1278. doi:10.1007/s00439-016-1720-4.

This article is protected by copyright. All rights reserved 